\title{
A NON-SEARCH OPTIMAL CONTROL SOLUTION FOR A TEAM OF MUAVS IN A RECONNAISSANCE MISSION
}

\author{
David W. Casbeer, Pengcheng Zhan, and A. Lee Swindlehurst \\ Dept. of Elec. \& Comp. Engineering, Brigham Young University, Provo, UT, USA, 84602 \\ e-mail:casbeer@byu.edu,pz8@ee.byu.edu,swindle@ee.byu.edu
}

\begin{abstract}
We consider a team of miniature unmanned air vehicles (MUAVs) in a multi-static radar scenario. Time delay and Doppler measurements made at the UAVs are transmitted to a base station which is tracking a target. The base then transmits heading commands to the MUAVs to reduce the tracking error. Optimal solutions that attempt to minimize a function of the error covariance or maximize the observability of the system are computationally difficult to implement. We present a simpler approximate method that yields a closed-form solution and performs comparably to the optimal approaches.
\end{abstract}

\section{INTRODUCTION}

Over the past decade, Unmanned Air Vehicles (UAVs) have moved into the spotlight as solutions for many military and civilian problems (e.g., surveillance, search and rescue, and tracking). This is in part due to technological advancements in batteries, sensors, and micro-controllers that make the UAVs more dependable and practical. Due to their versatility and cost, the "mini-UAV" (MUAV) class of aircraft is an increasing focus of research [1]-[3]. Because of their size, they are less apt to be detected, can fly at lower altitudes (obtaining more precise or localized information), and are easily launched, often by hand and without a runway.

In this paper we will investigate the use of a team of MUAVs for target tracking. We are particularly interested in coordinating their motion in order to improve tracking performance. Manipulating the trajectory of an individual airborne sensor taking bearing-only measurements has been considered in [4]-[6]. In recent years this theory has been applied to teams of UAVs where each agent is equipped with radar sensing equipment. In [7], an interesting information theoretic approach was implemented, while [8], [9] followed the traditional observability criteria developed by Kalman to improve the tracking performance through trajectory planning. Recently, [10] added the use of range measurements to this tracking problem.

The use of bearing measurements is practical on large sensor platforms. However, because MUAVs are small, they have a very limited aperture and they are more susceptible to turbulence than a larger UAV. Furthermore, their payload capacity as well as cost constraints inhibit the use of precisely accurate attitude sensors. Consequently, it is typically impractical to obtain reliable bearing measurements from a MUAV. Instead, in this paper, we examine the use of less sensitive Doppler and time delay measurements made by the MUAVs for target tracking. In an earlier paper [11], we presented two methods based on time-delay and Doppler that require gradient-search optimization of a complicated criterion function. Here we find an approximation that admits a closed-form solution, and that performs comparably to the optimal solution.

This research was supported by the National Science Foundation under Information Technology Research Grant CCR-0313056.

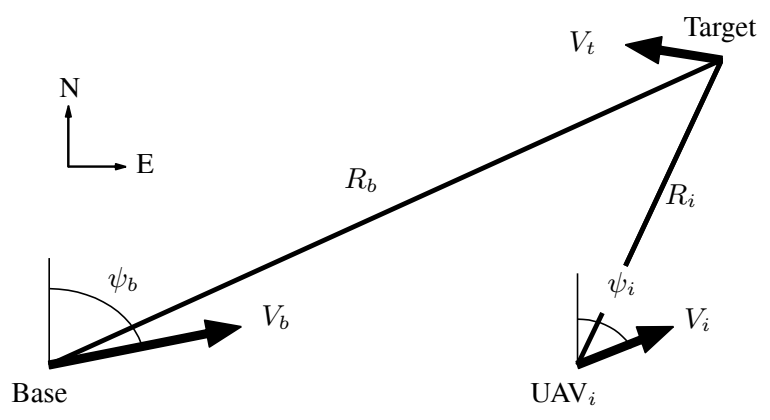

Fig. 1. Bistatic Radar scenario. Heading is measured wrt North.

\section{PROBLEM STATEMENT}

Figure 1 shows the multi-static radar setting we consider, where $N$ MUAVs make time-delay and Doppler measurements of a target based on a radar signal transmitted from a base. The MUAVs communicate their measurements to the base, which tracks the target and sends heading commands back to the MUAVs. The target state is written as $\mathbf{x}_{t}=\left[x_{t}, y_{t}, v_{t x}, v_{t y}\right]^{T}$, and consists of the target's $(x, y)$ position and velocity coordinates.

For the $i^{\text {th }}$ MUAV, the time delay and Doppler are given by

$$
\begin{aligned}
\tau_{i} & =\frac{1}{c}\left(R_{b}+R_{i}\right) \\
\omega_{i} & =-\omega_{c}\left(\frac{\partial R_{b}}{\partial t}+\frac{\partial R_{i}}{\partial t}\right),
\end{aligned}
$$

where $\omega_{c}$ is the radar carrier frequency, $c$ is the speed of light, and $R_{b}, R_{i}$ are respectively the distances from the base station to the target, and the target to MUAV $i$ [12]. Each MUAV is assumed to be equipped with an autopilot as described by [1]. The autopilot is capable of holding constant velocity and altitude, and is tuned to exhibit first order response to heading commands. The equations of motion are given by

$$
\begin{aligned}
\dot{x}_{i} & =V_{i} \cos \psi_{i}+w_{x} \\
\dot{y}_{i} & =V_{i} \sin \psi_{i}+w_{y} \\
\dot{\psi}_{i} & =\alpha_{\psi}\left(\psi_{i}^{c}-\psi_{i}\right)
\end{aligned}
$$

where $\mathbf{x}_{i}=\left[x_{i}, y_{i}\right]^{T}$ is the inertial position of the $i^{t h}$ MUAV, $\psi_{i}$ and $\psi_{i}^{c}$ are respectively its current and commanded heading, $V_{i}$ is the speed of the MUAV, $\mathbf{w}=\left[w_{x}, w_{y}\right]^{T}$ is the wind speed, and $\alpha_{\psi}$ is a parameter defining the response of the MUAV to heading commands.

\section{II-A. Extended Kalman Filter}

At each time step, we assume the base station receives measurements from all $N$ MUAVs, given by Equations (1) and (2). We 
stack these readings into the received vector

$$
\begin{aligned}
\mathbf{y}_{k} & =\mathbf{h}_{k}\left(\mathbf{x}_{t, k}\right)+v_{k} \\
& =\left[\tau_{1, k}, \cdots \tau_{N, k}, \omega_{1, k}, \cdots \omega_{N, k}\right]^{T}+v_{k}
\end{aligned}
$$

where the subscript $k$ has been added to denote the time associated with the data, and $v_{k}$ represents Gaussian measurement noise: The Jacobian of the received measurements will be written as $\mathbf{H}_{k}=$ $\left.\frac{\partial \mathbf{h}_{k}}{\partial \mathbf{x}}\right|_{\mathbf{x}_{k \mid k-1}}$. Assuming a nominal constant velocity model for the target, we write the propagation of the target's states as

$$
\mathbf{x}_{t, k}=\mathbf{A} \mathbf{x}_{t, k-1}+\eta_{k}=\left[\begin{array}{cccc}
1 & 0 & \Delta_{t} & 0 \\
0 & 1 & 0 & \Delta_{t} \\
0 & 0 & 1 & 0 \\
0 & 0 & 0 & 1
\end{array}\right] \mathbf{x}_{t, k-1}+\eta_{k}
$$

where $\eta_{k} \sim \mathcal{N}\left(\mathbf{0}, \mathbf{Q}_{k}\right)$ represents process noise.

Given the above, the Extended Kalman Filter (EKF) equations for the problem are given below:

- Time Update equations:

$$
\begin{aligned}
\mathbf{x}_{k \mid k-1} & =\mathbf{A} \mathbf{x}_{k-1 \mid k-1} \\
\mathbf{P}_{k \mid k-1} & =\mathbf{A} \mathbf{P}_{k-1 \mid k-1} \mathbf{A}^{T}+\mathbf{Q}_{k}
\end{aligned}
$$

- Measurement Update:

$$
\begin{aligned}
K_{k} & =\mathbf{P}_{k \mid k-1} \mathbf{H}_{k}^{T}\left(\mathbf{H}_{k} \mathbf{P}_{k \mid k-1} \mathbf{H}_{k}^{T}+\mathbf{R}_{k}\right)^{-1} \\
\mathbf{x}_{k \mid k} & =\mathbf{x}_{k \mid k-1}+K_{k}\left(\mathbf{y}_{k}-\mathbf{h}_{k}\left(\mathbf{x}_{k \mid k-1}\right)\right) \\
\mathbf{P}_{k \mid k} & =\left(\mathbf{I}-K_{k} \mathbf{H}_{k}\right) \mathbf{P}_{k \mid k-1}
\end{aligned}
$$

where $\mathbf{P}_{k \mid k-1}$ is the covariance of the state estimate for time $k$ given information up to time $k-1$. We desire to minimize the error in our state estimate. The reconfigurable nature of the sensor array enters into the Kalman filter through the Jacobian $\mathbf{H}_{k}$, and the MUAVs' motion will only effect future estimates of the state through measurement update equations (8)-(10). We will now discuss methods for controlling the MUAVs to improve our state estimate in this EKF scenario.

\section{OPTIMIZATION METHODS}

In this section, we describe three one-step optimization methods for adjusting the MUAV headings to optimally track the target according to various criteria.

\section{III-A. EKF Error Covariance Minimization}

In the first technique, we minimize a function of the EKF error covariance [11]. The optimal control for the MUAV team is found by propagating the error covariance $\mathbf{P}_{k \mid k}$ one step into the future using an estimate of the future measurements, and then minimizing the trace of the resulting $\hat{\mathbf{P}}_{k+1 \mid k+1}$. A $\hat{\bullet}$ has been used to emphasize that this is an estimated covariance since we do not know $\mathbf{H}_{k+1}$ at time $k$.

In particular, $\mathbf{P}_{k+1 \mid k}$ is calculated using equation (7), and we then assume acquisition of measurements at time $k+1$, given the target's estimated state $\mathbf{x}_{k+1 \mid k}$ and the propagated positions of the MUAV team using the vector heading for all MUAVs

$$
\psi_{k}=\left[\psi_{1} \cdots \psi_{N}\right]^{T} .
$$

We linearize about this estimated measurement and denote the resulting Jacobian by $\hat{\mathbf{H}}_{k+1}$. Then using equation (10), we estimate the error covariance at time $k+1$ and denote it by $\hat{\mathbf{P}}_{k+1 \mid k+1}$. The optimal MUAV headings are then given

$$
\psi_{k}^{c}=\underset{\psi_{k}}{\arg \min } \operatorname{trace}\left(\hat{\mathbf{P}}_{k+1 \mid k+1}\right) .
$$

\section{III-B. Information Filter Approach}

An alternative approach is to examine the equivalent information filter [13], where rather than propagating the state estimate error covariance, its inverse (i.e., the information matrix) is propagated. In this filtering scheme, upon acquiring new measurements, the state information matrix is updated as

$$
\mathbf{Y}_{k \mid k}=\mathbf{P}_{k \mid k}^{-1}=\mathbf{Y}_{k \mid k-1}+\mathbf{H}_{k}^{T} \mathbf{R}_{k}^{-1} \mathbf{H}_{k}
$$

where the term $\mathbf{H}_{k}^{T} \mathbf{R}_{k}^{-1} \mathbf{H}_{k}$ accounts for an information increase in the state estimate due to the new observations. Here we desire to maximize the information received in the next observation at time $k+1$.

A simple approximation to maximize the received information is made by setting the process noise to zero: $\mathbf{Q}=0$. Then, to maximize the information we simply maximize the determinant of the received information matrix [10], [14]:

$$
\mathscr{I}_{k+n, k}=\sum_{i=k}^{k+n} \Phi_{i, k+n}^{T} \hat{\mathbf{H}}_{i}^{T} \mathbf{R}_{i}^{-1} \hat{\mathbf{H}}_{i} \Phi_{i, k+n}
$$

where $\Phi_{t_{f}, t_{0}}$ is the state transition matrix from time $t_{0}$ to $t_{f}$. This matrix accounts for the loss or gain in information due to the motion of the target, which can be seen from equation (7). Under the assumptions in this paper, $\Phi_{t_{f}, t_{0}}=\mathbf{A}^{t_{f}-t_{0}}$, and for one-step case $(n=1)$ the algorithm becomes

$$
\begin{aligned}
\psi_{k}^{c} & =\underset{\psi_{k}}{\arg \min \operatorname{det}}\left(\mathscr{I}_{k+1, k}\right) \\
& =\underset{\psi_{k}}{\arg \min \operatorname{det}}\left(\mathbf{A}^{-T} \hat{\mathbf{H}}_{k+1}^{T} \mathbf{R}_{k+1}^{-1} \hat{\mathbf{H}}_{k+1} \mathbf{A}^{-1}\right) .
\end{aligned}
$$

\section{III-C. Information Theoretic approach}

Mutual information $I(\mathbf{x} ; \mathbf{y})=H(\mathbf{x})-H(\mathbf{x} \mid \mathbf{y})$ can be viewed as the reduction in the uncertainty of $\mathbf{x}$ given knowledge of $\mathbf{y}$ [15]. We would like to maximize this reduction in uncertainty, or equivalently minimize the conditional entropy $H(\mathbf{x} \mid \mathbf{y})$ which contains the term we have control over using the MUAVs (y).

In order to determine the condition entropy we first linearize the observation received about the state estimate:

$$
\mathbf{y}(k+1) \approx \mathbf{h}\left(\hat{\mathbf{x}}_{k+1 \mid k}\right)+\mathbf{H}_{k}\left(\mathbf{x}_{k+1}-\hat{\mathbf{x}}_{k+1 \mid k}\right)+v_{k+1} .
$$

Then stacking the state and measurement errors together, we have

$$
\left[\begin{array}{c}
\mathbf{x}_{k}-\hat{\mathbf{x}}_{k+1 \mid k} \\
\mathbf{y}(k)-\mathbf{h}\left(\hat{\mathbf{x}}_{k+1 \mid k}\right)
\end{array}\right] \sim \mathcal{N}\left(\mathbf{0}, \boldsymbol{\Sigma}_{k+1 \mid k}\right)
$$

where

$$
\boldsymbol{\Sigma}_{k+1 \mid k}=\left[\begin{array}{cc}
\hat{\mathbf{P}}_{k+1 \mid k} & \hat{\mathbf{P}}_{k+1 \mid k} \hat{\mathbf{H}}_{k+1}^{T} \\
\hat{\mathbf{H}}_{k+1} \hat{\mathbf{P}}_{k+1 \mid k} & \hat{\mathbf{H}}_{k+1} \hat{\mathbf{P}}_{k+1 \mid k} \hat{\mathbf{H}}_{k+1}^{T}+\mathbf{R}_{k+1}
\end{array}\right] .
$$

The conditional entropy can be written as

$$
\begin{aligned}
H(\mathbf{x} \mid \mathbf{y}) & =H(\mathbf{x}, \mathbf{y})-H(\mathbf{y}) \\
& =\frac{1}{2} \log \left((2 \pi e)^{4} \frac{\left|\boldsymbol{\Sigma}_{k+1 \mid k}\right|}{\left|\hat{\mathbf{H}}_{k+1} \hat{\mathbf{P}}_{k+1 \mid k} \hat{\mathbf{H}}_{k+1}^{T}+\mathbf{R}_{k+1}\right|}\right) .
\end{aligned}
$$

In equation (14), the covariance term in the denominator is similar to the observability matrix given in (13). This term maximizes the information gathered from the observations. However the joint covariance $\boldsymbol{\Sigma}_{k+1 \mid k}$ in the numerator is added and accounts for the fact that we not only want to maximize the new information but that it be as different as possible from what we already know about the state. Formally the optimal heading commands are found by

$$
\psi_{k}^{c}=\underset{\psi_{k}}{\arg \min } H\left(\mathbf{x}_{k+1 \mid k} \mid \mathbf{y}_{k+1}\right) .
$$




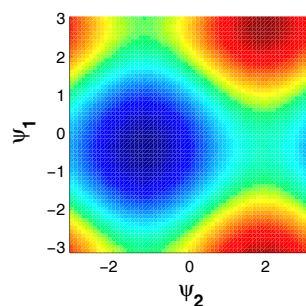

(a) Cost function

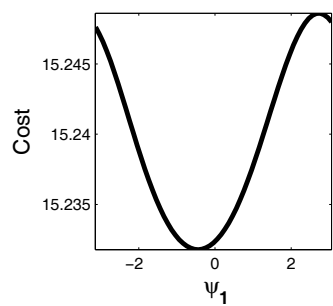

(b) Cost function: $\psi_{2}$ is set to previous heading
Fig. 2. One realization of observability matrix plotted against $\psi_{1}$ and $\psi_{2}$, where $n=10$ in equation (13).

\section{CLOSED FORM SOLUTION}

Using $\Delta_{t}$ to denote the time step, we note that the $i^{t h}$ MUAV's position on step in the future can be approximated by

$$
\begin{aligned}
x_{i}(k+1) & \approx x_{i}(k)+\Delta_{t} V_{i} \cos \psi_{i} \\
y_{i}(k+1) & \approx y_{i}(k)+\Delta_{t} V_{i} \sin \psi_{i} .
\end{aligned}
$$

This implies the MUAVs position, as a function of heading, is sinusoidal in nature. Figure 2(a) depicts the surface of the cost function in equation (13) in Section III-B. Figure 2(b) is a slice from the 3-D surface on the left, found by setting $\psi_{2}=0$. (The surfaces for the estimated covariance matrix and conditional entropy, given the same MUAV and target parameters, have also been shown at the end of the paper (see Fig. 6))

Given this observation, we approximate our cost function as sinusoidal, and assume that it is separable in the various MUAV headings (i.e., each MUAV's heading depends on the others only in amplitude, denoted by $\alpha$ ). We assume that the MUAV's heading is dependent on the target's state through a phase parameter we label $\theta$. More specifically, we model our cost function as a sinusoid with a known frequency and unknown amplitude $(\alpha)$ and phase $(\theta)$, corrupted by iid gaussian noise, $\gamma\left(\psi_{i}\right)$ :

$$
J\left(\psi_{i}\right)=\alpha \cos \left(\psi_{i}+\theta\right)+\beta+\gamma\left(\psi_{i}\right),
$$

The constant $\beta$ is independent of $\theta$; therefore it does not effect the ML estimate and will be dropped in the sequel. In finding the heading for MUAV $i$, we set the heading for all MUAVs $j \neq i$ to their previous values. We need only specify the phase term in order to completely approximate the criterion function. The maximum likelihood phase estimate is given by

$$
\tan \left(\hat{\theta}_{M L}\right)=-\frac{\int_{0}^{T} J\left(t_{1}\right) \sin \left(t_{1}\right) d t_{1}}{\int_{0}^{T} J\left(t_{2}\right) \cos \left(t_{2}\right) d t_{2}} .
$$

We approximate the integrals by finite summations:

$$
\begin{aligned}
\tan \left(\hat{\theta}_{M L}\right) \approx & -\frac{\sum_{t_{1}} J\left(t_{1}\right) \sin \left(t_{1}\right)}{\sum_{t_{2}} J\left(t_{2}\right) \cos \left(t_{2}\right)} \\
& \forall t_{1}, t_{2}=\frac{j 2 \pi}{m}, \quad j \in[0, m-1] .
\end{aligned}
$$

In the simulations of Section $\mathrm{V}$, the use of the four samples $t_{1}, t_{2}=$ $\left\{0, \frac{\pi}{2}, \pi, \frac{3 \pi}{2}\right\}$ is shown to give good performance. Notice that this approach decouples estimation of the MUAV heading angles due to the cancellation of $\alpha$ in equation (17). Using the arctan function to determine $\theta_{M L}$, the minimum and maximum values of the cost function will be approximately located at:

$$
\begin{aligned}
\psi_{\min } & =\pi-\theta_{M L} \\
\psi_{\max } & =-\theta_{M L},
\end{aligned}
$$
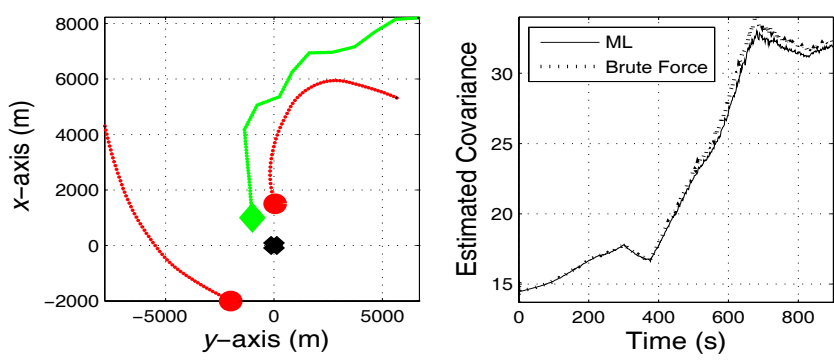

Fig. 3. Trajectories on left. Corresponding estimated covariance $\left.\operatorname{trace}\left(\hat{\mathbf{P}}_{k+1 \mid k+1}\right)\right)$ for ML and brute force search on left.
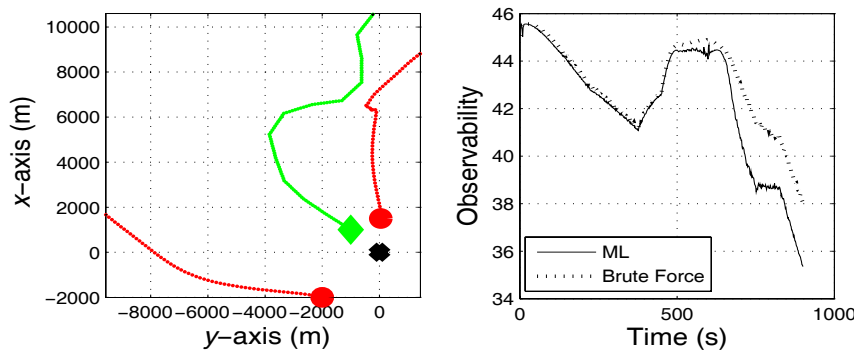

Fig. 4. Trajectories on left. Corresponding observability $\left(\left|\mathscr{I}_{k+1, k}\right|\right)$ for ML and brute force search on left.

where we will use either min or max depending on the cost function being tested.

\section{SIMULATION RESULTS}

For all simulations, distance will be measured in meters, and each simulation runs for a period of 900s. The base station is assumed to be located at the the origin, and is denoted by a thick black dot in the figures. The UAVs are initially located at at $(1500,50) \mathrm{m}$ and $(-2000,-2000) \mathrm{m}$, and are shown as large circles in the plots. The MUAVs travel with a constant speed of $10 \mathrm{~m} / \mathrm{s}$, while the target moves with a nominal (undisturbed) speed $20 \mathrm{~m} / \mathrm{s}$ originating at $(1000,-1000) \mathrm{m}$. The target is depicted using a large diamond. The measurement and process noise in the Kalman filter are assumed to be constant, and are set to be a scaled identity matrix: $\mathbf{Q}=\mathbf{R}=10 \mathbf{I}$.

Figures 3, 4 and 5 illustrate the effectiveness of the ML phase estimation minimization technique (Section IV). In these figures, the three cost functions described in Section III have been employed. A brute force optimization has been employed, as well as the ML approximation for each cost. The difference in trajectories between the brute force and ML optimization was minimal for each cost; therefore,we have only plotted one instance of their flight paths. The difference between the optimization techniques for each cost function can be seen in the right subfigures of Figures 3, 4 and 5. We can see that the ML approximation performed close to the optimal solution for these scenarios.

Using the three cost functions described in this paper, we ran Monte Carlo simulations placing the target and MUAVs randomly in a $1000 \times 1000 \mathrm{~m}^{2}$ area, assigning each a random heading. It was found that in $12 \%$ of the cases, the approximate method failed to give performance similar to direct minimization of the criteria. This failure rate was consistent among all three cost functions. Typically, the approximate closed-form solution failed in cases where the MUAVs' geometry causes $\mathbf{H}^{T} \mathbf{H}$ to become low rank (i.e., non-diverse measurements). In our measurement model, this 

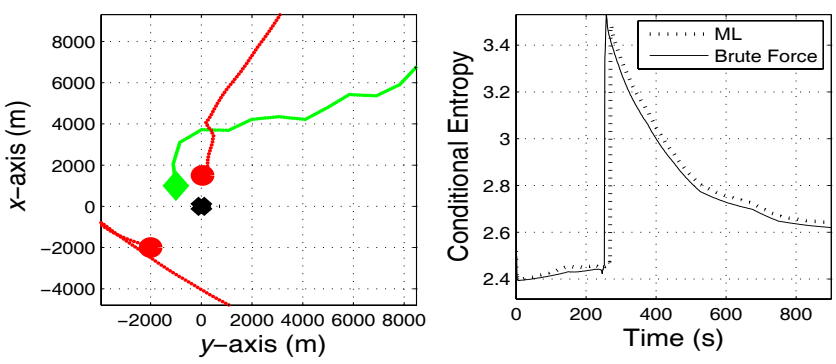

Fig. 5. Trajectories on left. Corresponding conditional entropy $(H(\mathbf{x} \mid \mathbf{y}))$ for ML and brute force search on left.

can occur at two times: when the MUAVs approach each other or when they are positioned approximately symmetrically about the velocity vector of the target. In most scenarios, such situations can be easily avoided, and the approximate solution will perform well.

\section{CONCLUSIONS AND FUTURE WORK}

In this paper we have presented a closed-form solution to the problem of finding the optimal trajectory for a team of MUAVs involved in target tracking. We considered three different optimization approaches to the problem, and found that the approximation worked well in most cases for all three. Simulations show that the closed-form solution closely tracks the performance of a full brute-force optimization for realistic tracking scenarios.

\section{REFERENCES}

[1] Randal et al. Beard, "Autonomous vehicle technologies for small fixed wing uavs," AIAA Journal of Aerospace Computing, Information, and Communication, vol. 2, no. 1, pp. 92-108, 2005.

[2] R. Beard, T. McLain, D. Nelson, and D. Kingston, "Decentralized cooperative aerial surveillance using fixed-wing miniature uavs," IEEE Proceedings: Special Issue on Mulit-Robot Systmes, (to appear) Technical Report available at https://dspace.byu.edu/handle/1877/60.

[3] D.W. Casbeer and et al., "Cooperative forest fire surveillance using a team of small unmanned air vehicles," International Journal of Systems Sciences, (to appear) Technical Report available at https://dspace.byu.edu/handle/1877/55.

[4] V.J. Aidala, "Kalman filter behavior in bearings-only tracking applications," IEEE Trans. Aerosp. Electron. Syst., pp. 29-39, Jan. 1979.

[5] S.C. Nardone, A.G. Lindgren, and K.F. Gong, "Fundamental properties and performance of conventional bearings-only target motion analysis," IEEE Trans. Automat. Contr., vol. 29, no. 9, pp. 775-787, Sept. 1984.

[6] S.E. Hammel, P.T. Liu, E.J. Hilliard, and K.F. Gong, "Optimal observer motion for localization with bearing measurements," Computers and Mathematics, With Applications, vol. 18, no. 3, pp. 171-180, 1989.

[7] Marcel L. Hernandez, "Optimal sensor trajectories in bearings-only tracking," in Proceedings of the Seventh International Conference on Information Fusion, Mountain View, CA, Jun 2004, vol. II, pp. 893-900, International Society of Information Fusion.

[8] Y. Oshman and P. Davidson, "Optimization of observer trajectories for bearings-only target localization,” IEEE Trans. Aerosp. Electron. Syst., vol. 35, no. 3, pp. 892-902, July 1999.

[9] B. Grocholsky, A. Makarenko, and H. Durrant-Whyte, "Informationtheoretic coordinated control of multiple sensor platforms," in Proceedings of the IEEE International Conference on Robotics and Automation, 2003, vol. 1, pp. 1521-1526.

[10] J. Ousingsawat and M. Campbell, "Establishing trajectories for multivehicle reconnaissance," in Proceedings of the AIAA Guidance, Navigation, and Control Conference, Aug. 2004, pp. 2188-2199.

[11] Pengcheng Zhan, David Casbeer, and A.Lee Swindlehurst, "A centralized control algorithm for target tracking with uavs," in Proc. 39th Asilomar Conf., Pacific Grove, CA, October 2005.

[12] N. J. Willis, Bistatic Radar, Artech House, Boston, 1991.
[13] Arthur Mutambara, Decentralized Estimation and Control for Multisensor Systems, CRC Press LLC, Boca Raton, Florida, 1998.

[14] Andrew H. Jazwinski, Stochastic Processes and Filtering Theory, vol. 64 of Mathematics in Science and Engineering, Academic Press, Ney York, 1970.

[15] Thomas M. Cover and Joy A. Thomas, Elements of Information Theory, John Wiley \& Sons, Inc., N. Y., 1991.

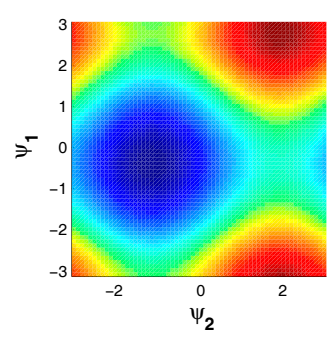

(a) Estimated Covariance

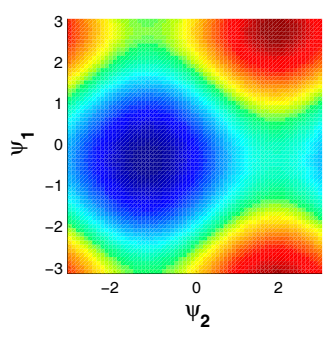

(b) Conditional Entropy
Fig. 6. Surfaces of cost functions plotted against $\psi_{1}$ and $\psi_{2}$. Compare with Fig. 2. 